\title{
Application of the Asymptotic Taylor Expansion Method to Bistable Potentials
}

\author{
Okan Ozer, Halide Koklu, and Serap Resitoglu \\ Department of Engineering Physics, Faculty of Engineering, University of Gaziantep, 27310 Gaziantep, Turkey \\ Correspondence should be addressed to Okan Ozer; ozer@gantep.edu.tr
}

Received 15 April 2013; Accepted 15 July 2013

Academic Editor: Fedele Lizzi

Copyright (C) 2013 Okan Ozer et al. This is an open access article distributed under the Creative Commons Attribution License, which permits unrestricted use, distribution, and reproduction in any medium, provided the original work is properly cited.

A recent method called asymptotic Taylor expansion (ATEM) is applied to determine the analytical expression for eigenfunctions and numerical results for eigenvalues of the Schrödinger equation for the bistable potentials. Optimal truncation of the Taylor series gives a best possible analytical expression for eigenfunctions and numerical results for eigenvalues. It is shown that the results are obtained by a simple algorithm constructed for a computer system using symbolic or numerical calculation. It is observed that ATEM produces excellent results consistent with the existing literature.

\section{Introduction}

There is no doubt that an interesting problem in fundamental quantum mechanics for lecturers and advanced undergraduate and graduate students in physics and applied mathematics is to obtain the exact solutions of the Schrödinger equation for any type of potential. It is well known that the Schrödinger equation, proposed by Erwin Schrödinger in 1926, is a second-order differential equation that describes how the quantum state of a physical system changes with time [1]. It is as central to quantum mechanics as Newton's laws are to classical mechanics. It is also well known that the quantum mechanics is established on some certain postulates, and in any introductory quantum physics textbook these postulates can be found with the application of the time-independent Schrödinger equation for a single particle in one dimension as a simple and basic example.

Unfortunately, there are not so many potentials that can be solved exactly such as the Coulomb, harmonic oscillator, and Pöschl-Teller potentials. Since one of the source of progress of the science depends on the study of the same problem from different point of view, various methods have been suggested such as numerical calculation $[2,3]$, the variational $[2,4]$, the perturbation [5], the WKB $[6,7]$, the shifted $1 / N$ expansion $[8,9]$, the Nikiforov-Uvarov (NU) $[10,11]$, the supersymmetry (SUSY) [12,13], the generalized pseudospectral (GPS) [14], the asymptotic iteration method (AIM) [15], and other methods [16-21] to find the approximate solutions of the potentials that are not exactly solvable.

In this study we will apply a new formalism based on the Taylor series expansion method, namely, asymptotic Taylor expansion method (ATEM) [22], to bistable potentials. These type of potentials have been used in the quantum theory of molecules as a crude model to describe the motion of a particle in the presence of two centers of force [23-29]. It is mentioned in [22] that the taylor series Method [30, 31] is an old one but it has not been fully exploited in the analysis of both physical and mathematical problems in solution. It is also claimed that ATEM can also be easily applied to solve second-order differential equations by introducing a simple Mathematica [32] computer program. Therefore, we focus on the solution of the eigenvalue problems of some type of bistable potentials by using ATEM in this paper.

The organization of the paper is as follows. In Section 2, we present a brief outline of the method ATEM. In Section 3, the eigenvalues of the bistable potentials are determined by using ATEM. Section 4 is devoted to a conclusion.

\section{Formalism of Asymptotic Taylor Expansion Method}

In this section, we present the solution of the Schrödingertype equations by modifying Taylor series expansion with the aid of a finite sequence instead of an infinite sequence and its 
termination possessing the property of quantum mechanical wave function. It is well known in quantum mechanics that the bound-state energy of an atom is quantized and eigenvalues are discrete. Additionally, for each eigenvalues there exist one or more eigenfunctions. If one considers the solution of the Schrödinger equation, discrete eigenvalues of the problem are mainly under investigation. The first main result of this conclusion gives necessary and sufficient conditions for the termination of the Taylor series expansion of the wave function.

Following the notation in [22], one can consider the Taylor series expansion of a function $f(x)$ about the point $a$ :

$$
\begin{aligned}
f(x)= & f(a)+(x-a) f^{\prime}(a)+\frac{1}{2}(x-a)^{2} f^{\prime \prime}(a) \\
& +\frac{1}{6}(x-a)^{3} f^{(3)}(a)+\cdots \\
= & \sum_{n=0}^{\infty} \frac{(x-a)^{n}}{n !} f^{(n)}(a),
\end{aligned}
$$

where $f^{(n)}(a)$ is the $n$th derivative of the function at $a$. Taylor series specifies the value of a function at one point, $x$, in terms of the value of the function and its derivatives at a reference point $a$. Expansion of the function $f(x)$ about the origin $(a=$ 0 ) is known as Maclaurin's series and it is given by

$$
\begin{aligned}
f(x)= & f(0)+x f^{\prime}(0)+\frac{1}{2} x^{2} f^{\prime \prime}(0) \\
& +\frac{1}{6} x^{3} f^{(3)}(0)+\cdots \\
= & \sum_{n=0}^{\infty} \frac{x^{n}}{n !} f^{(n)}(0) .
\end{aligned}
$$

Here one can develop a method to solve a second-order linear differential equation of the form

$$
f^{\prime \prime}(x)=p_{0}(x) f^{\prime}(x)+q_{0}(x) f(x) .
$$

It is seen that the higher-order derivatives of the $f(x)$ can be obtained in terms of the $f(x)$ and $f^{\prime}(x)$ by differentiating (3). Then, higher-order derivatives of $f(x)$ are given by

$$
f^{(n+2)}(x)=p_{n}(x) f^{\prime}(x)+q_{n}(x) f(x),
$$

where

$$
\begin{gathered}
p_{n}(x)=p_{0}(x) p_{n-1}(x)+p_{n-1}^{\prime}(x)+q_{n-1}(x), \\
q_{n}(x)=q_{0}(x) p_{n-1}(x)+q_{n-1}^{\prime}(x) .
\end{gathered}
$$

At this point, one can observe that the eigenvalues and eigenfunctions of the Schrödinger-type equations can efficiently be determined by using ATEM. To this end, the recurrence relations (5) allow one to determine algebraic exact or approximate analytical solution of (3) under some certain conditions. Let us now substitute (5) into (1) to obtain the function that is related to the wave function of the corresponding Hamiltonian:

$$
\begin{aligned}
f(x)= & f(0)\left(1+\sum_{n=2}^{m} q_{n-2}(0) \frac{x^{n}}{n !}\right) \\
& +f^{\prime}(0)\left(1+\sum_{n=2}^{m} p_{n-2}(0) \frac{x^{n}}{n !}\right) .
\end{aligned}
$$

After all, one can now obtain useful formalism of the Taylor expansion method. This form of the Taylor series can also be used to obtain series solution of the second-order differential equations. In the solution of the eigenvalue problems, truncation of the asymptotic expansion to a finite number of terms is useful. If the series optimally truncated at the smallest term then the asymptotic expansion of series is known as superasymptotic [33], and it leads to the determination of eigenvalues with minimum error.

Since the improper sets of boundary conditions may produce nonphysical results, arrangement of the boundary conditions for different problems becomes very important. When only odd or even power of $x$ is collected as coefficients of $f(0)$ or $f^{\prime}(0)$ and vice versa, the series is truncated at $n=m$; then an immediate practical consequence of these conditions is obtained for $q_{m-2}(0)=0$ or $p_{m-2}(0)=0$. In this way, the series truncates at $n=m$ and one of the parameters in the $q_{m-2}(0)$ or $p_{m-2}(0)$ belongs to the spectrum of the Schrödinger equation. Therefore eigenfunction of the equation becomes a polynomial of degree $m$. Otherwise the spectrum of the system can be obtained as follows. In a quantum mechanical system eigenfunction of the system is discrete. Therefore in order to terminate the eigenfunction $f(x)$ we can concisely write that

$$
\begin{gathered}
q_{m}(0) f(0)+p_{m}(0) f^{\prime}(0)=0, \\
q_{m-1}(0) f(0)+p_{m-1}(0) f^{\prime}(0)=0 .
\end{gathered}
$$

Eliminating $f(0)$ and $f^{\prime}(0)$ we obtain

$$
q_{m}(0) p_{m-1}(0)-p_{m}(0) q_{m-1}(0)=0
$$

again one of the parameters in the equation related to the eigenvalues of the problem.

It has been stated that the ATEM reproduces exact solutions to many exactly solvable differential equations and these equations can be related to the Schrödinger equation. It is observed that the process presented in ATEM is iterative and the number of iteration is given by $m$. The method can be applied to the Schrödinger equation with any type of potential as follows. Using a computer program, one first sets up the iteration number $m$, say $m=30$, to obtain the result. Then, setting $m=40$, another result is obtained. This procedure is repeated for different $m$ values leading to different results. Finally, one can compare the results for each case till desired digits. If the values of the eigenvalue reach their asymptotic values, then one can choose the corresponding $m$ value and truncate the iteration for next calculations. For instance, if one can obtain the values of the eigenvalues for $m=60$, first 


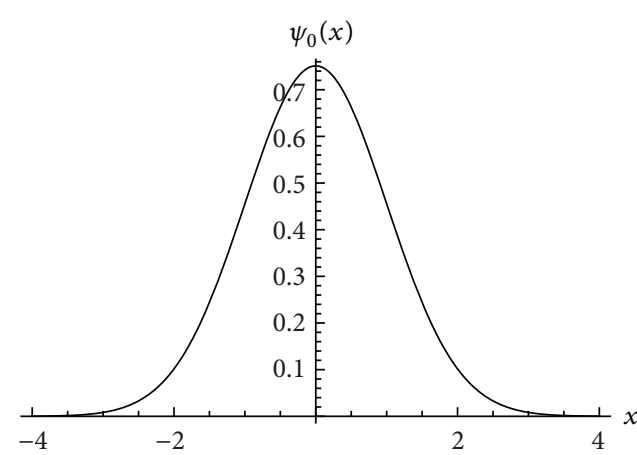

(a)

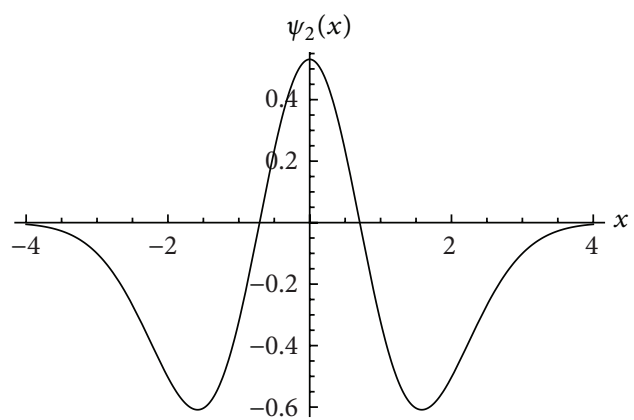

(c)

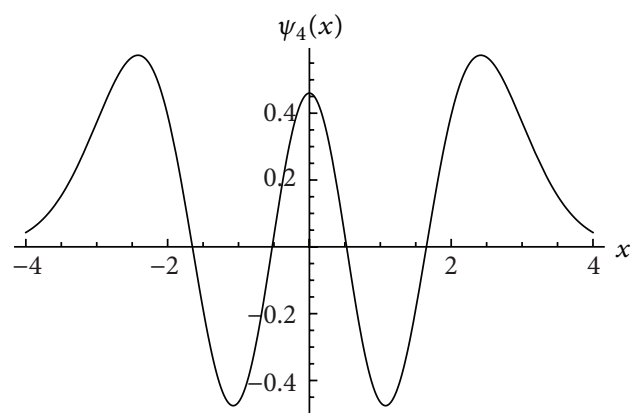

(e)

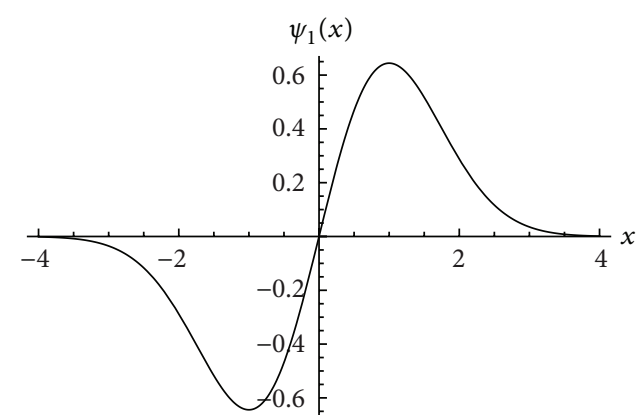

(b)

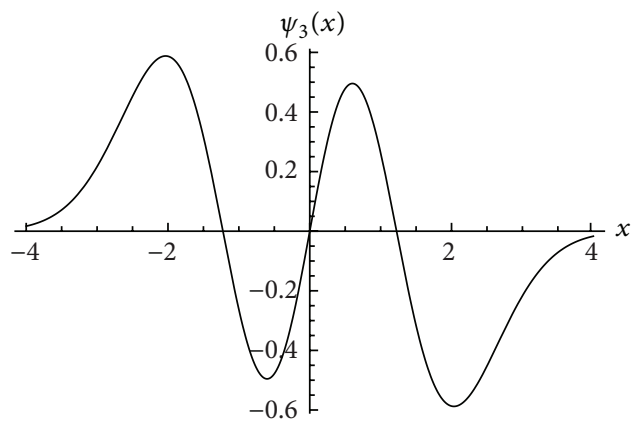

(d)

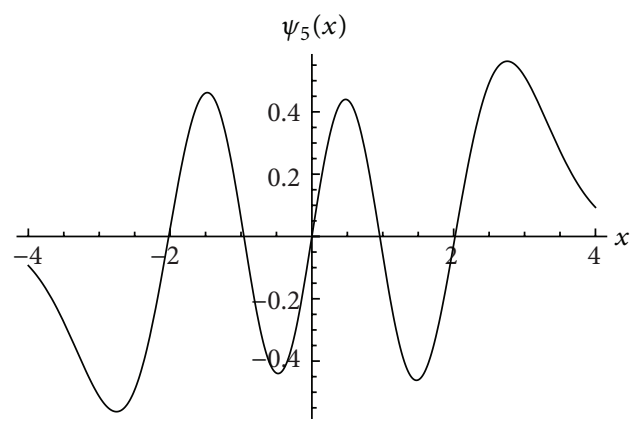

(f)

FIgURE 1: The first six states normalized eigenfunctions of the Harmonic potential given in (10).

few of them - first eight eigenvalues, for example-will reach automatically their asymptotic values. The following comment on the function is considerable. For such a solution it may be suitable to take sum of the first eight terms in (6).

\section{Applications}

We shall illustrate here that (3) with conditions (7) and (8) gives a complete solution for some important Schrödingertype problems. Through a concrete example we explore the solution of Schrödinger equation $\left(\hbar^{2}=2 m=1\right)$ :

$$
\left(-\frac{d^{2}}{d x^{2}}+V(x)\right) \psi(x)=E \psi(x)
$$

for the harmonic oscillator potential in one dimension $V(x)=x^{2}$ given as

$$
\left(-\frac{d^{2}}{d x^{2}}+x^{2}\right) \psi(x)=E \psi(x)
$$

In the limit of large $x$, the asymptotic solutions of (10) can be taken as any power of $x$ times a decreasing Gaussian function to satisfy the quantum mechanics postulates. With this in mind, one can write the "unnormalized" wave functions as

$$
\psi(x)=\exp \left(-\frac{x^{2}}{2}\right) f(x)
$$


TABLE 1: Eigenvalues of the potential in (15) for a range of $g$ values. For ATEM results, number of iterations for each $g$ value is set to 120 . For SUSY results, numbers in parenthesis $(N)$ denote the perturbation order used by authors in [40].

\begin{tabular}{|c|c|c|c|}
\hline$g$ & $E_{\text {ATEM }}$ & $E_{\text {SUSY }}[40]$ & $E_{\text {exact }}[42]$ \\
\hline \multirow{4}{*}{0.01} & 1.007373 & $1.00737(N=4)$ & 1.007373 \\
\hline & 3.036525 & $3.03653(N=8)$ & 3.036525 \\
\hline & 5.093939 & $5.09609(N=15)$ & 5.093939 \\
\hline & 7.178573 & $7.19832(N=15)$ & 7.178573 \\
\hline \multirow{4}{*}{0.05} & 1.034729 & $1.03473(N=4)$ & 1.034729 \\
\hline & 3.167225 & $3.16723(N=8)$ & 3.167225 \\
\hline & 5.417261 & $5.42404(N=15)$ & 5.417261 \\
\hline & 7.770271 & $7.83995(N=15)$ & 7.770271 \\
\hline \multirow{4}{*}{0.1} & 1.065286 & $1.06528(N=4)$ & 1.065286 \\
\hline & 3.306872 & $3.30687(N=8)$ & 3.306872 \\
\hline & 5.747959 & $5.75694(N=15)$ & 5.747959 \\
\hline & 8.352678 & $8.45913(N=15)$ & 8.352678 \\
\hline \multirow{4}{*}{0.5} & 1.2418541 & $1.24118(N=4)$ & 1.2418541 \\
\hline & 4.051932 & $4.05171(N=8)$ & 4.051932 \\
\hline & 7.396900 & $7.40489(N=15)$ & 7.396900 \\
\hline & 11.11515 & $11.3415(N=15)$ & 11.11515 \\
\hline \multirow{4}{*}{1} & 1.392352 & $1.39017(N=4)$ & 1.392352 \\
\hline & 4.648813 & $4.64784(N=8)$ & 4.648813 \\
\hline & 8.655049 & $8.65908(N=15)$ & 8.655049 \\
\hline & 13.15680 & $13.4524(N=15)$ & 13.15680 \\
\hline \multirow{4}{*}{10} & 2.449174 & $2.42910(N=4)$ & 2.449174 \\
\hline & 8.599003 & $8.58582(N=8)$ & 8.599003 \\
\hline & 16.63592 & $16.6188(N=15)$ & 16.63592 \\
\hline & 25.80627 & $26.4698(N=15)$ & 25.80627 \\
\hline \multirow{4}{*}{100} & 4.999410 & $4.93770(N=4)$ & 4.999418 \\
\hline & 17.83000 & $17.7864(N=8)$ & 17.83019 \\
\hline & 34.87117 & $34.8238(N=15)$ & 34.87398 \\
\hline & 54.36576 & $55.4001(N=15)$ & 54.38529 \\
\hline
\end{tabular}

TABLE 2: The first ten eigenvalues of the potential in (15) for $g=0.1$. Number of iteration is set to $m=120$.

\begin{tabular}{lllllllllllll}
\hline$n$ & \multicolumn{9}{c}{$E_{\text {ATEM }}$} \\
\hline 0 & 1.065 & 285 & 509 & 543 & 717 & 701 & 1.065 & 285 & 509 & 543 & 717 & 688 \\
1 & 3.306 & 872 & 013 & 152 & 913 & 680 & 3.306 & 872 & 013 & 152 & 913 & 507 \\
2 & 5.747 & 959 & 268 & 833 & 563 & 228 & 5.147 & 959 & 268 & 833 & 563 & 304 \\
3 & 8.352 & 677 & 825 & 785 & 754 & 350 & 8.352 & 677 & 825 & 785 & 754 & 712 \\
4 & 11.098 & 595 & 622 & 633 & 043 & 333 & 11.098 & 595 & 622 & 633 & 043 & 011 \\
5 & 13.969 & 926 & 197 & 742 & 799 & 089 & 13.969 & 926 & 197 & 742 & 799 & 300 \\
6 & 16.954 & 794 & 686 & 144 & 150 & 972 & 16.954 & 794 & 686 & 144 & 151 & 337 \\
7 & 20.043 & 863 & 604 & 188 & 462 & 801 & 20.043 & 863 & 604 & 188 & 461 & 233 \\
8 & 23.229 & 552 & 179 & 939 & 290 & 112 & 23.229 & 552 & 179 & 939 & 289 & 070 \\
9 & 26.505 & 554 & 752 & 536 & 617 & 968 & 26.505 & 554 & 752 & 536 & 617 & 417 \\
\hline
\end{tabular}

where the functions $f(x)$ are to be found by means of the iteration procedure given previously. Substituting (11) into (10), one obtains

$$
L(x)=-f^{\prime \prime}(x)+2 x f^{\prime}(x)+(1-E) f(x)=0 .
$$

Comparing (3) and (12) one can deduce that

$$
p_{0}(x)=2 x, \quad q_{0}(x)=1-E .
$$

Following the procedure given in (8) yields the exact eigenvalues of the harmonic oscillator potential:

$$
E_{n}=2 n+1, \quad n=0,1,2,3, \ldots,
$$

and the normalized eigenfunctions of the Schrödinger equation in (10) are shown in Figure 1.

As a second application, we consider the quartic anharmonic oscillator potential that has been a great deal of interest 


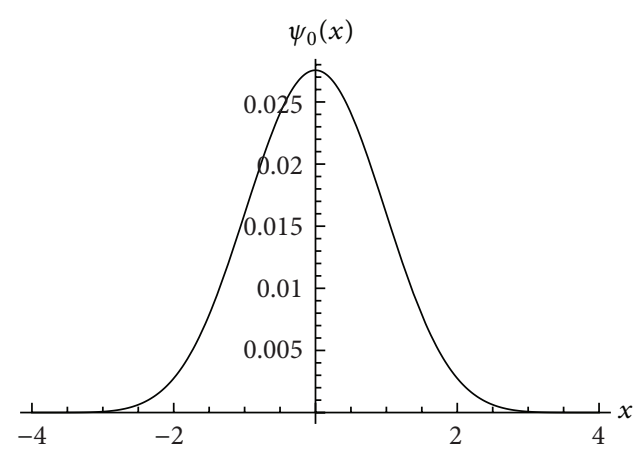

(a)

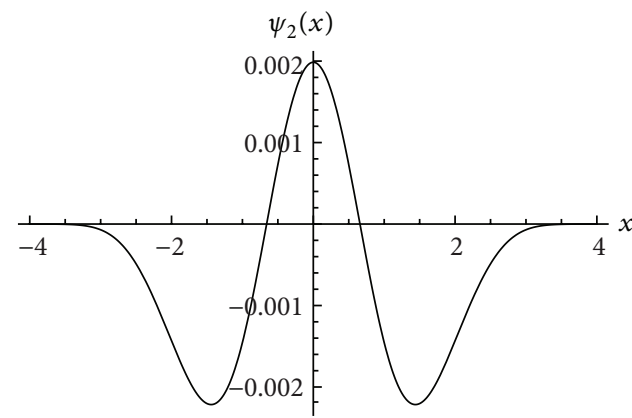

(c)

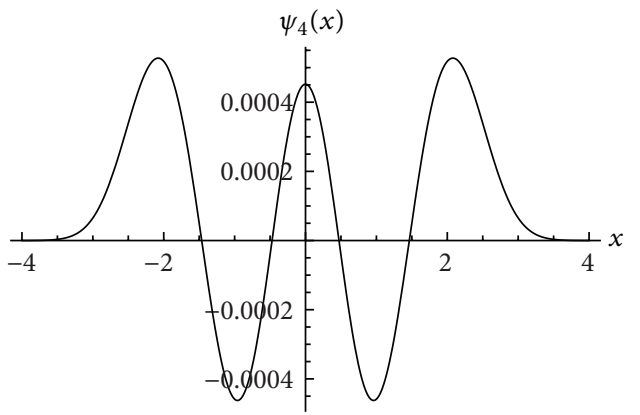

(e)

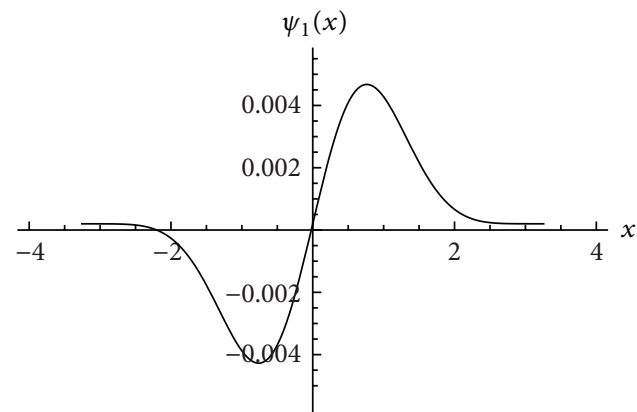

(b)

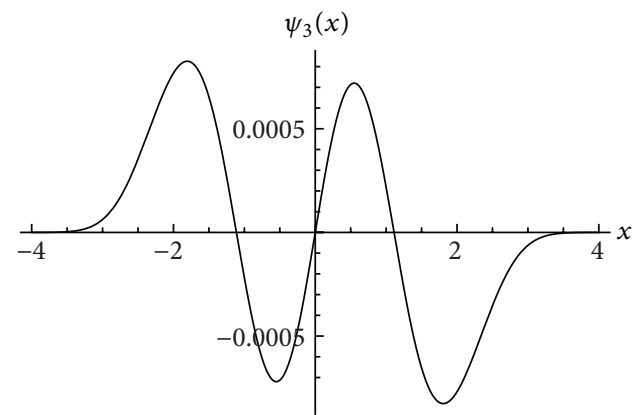

(d)

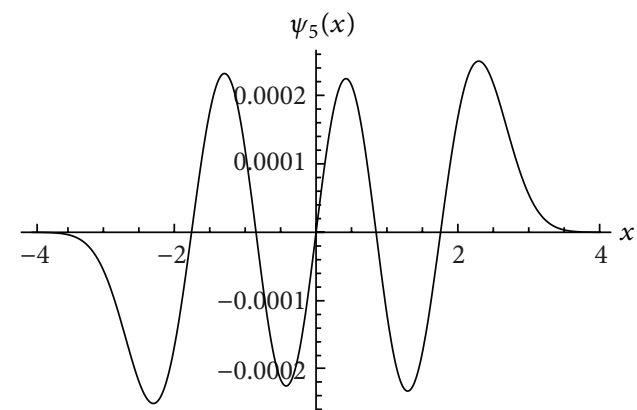

(f)

FIGURE 2: The first six states normalized eigenfunctions of the quartic potential given in (15) for $g=0.1$.

in the analytical and numerical investigations of the onedimensional anharmonic oscillators because of their importance in molecular vibrations [34] as well as in solid state physics [35, 36] and quantum field theories [37]. Schrödinger equation (9) for the one-dimensional quartic anharmonic potential $V(x)=x^{2}+g x^{4}$ is written as

$$
\left(-\frac{d^{2}}{d x^{2}}+x^{2}+g x^{4}\right) \psi(x)=E \psi(x),
$$

where $g>0$. The solution of (15) has been always studied to test accuracy and efficiency of the different methods proposed in $[15,38-41]$. Now, we introduce the asymptotic solutions of (15) as

$$
\psi(x)=e^{-\alpha\left(x^{2} / 2\right)-\beta\left(x^{4} / 4\right)} f(x),
$$

and (15) can now be written as

$$
\begin{aligned}
L(x)= & -f^{\prime \prime}(x)+2\left(\alpha x+\beta x^{3}\right) f^{\prime}(x) \\
& +\left(\alpha-E+\left(1-\alpha^{2}+3 \beta\right) x^{2}+(g-2 \alpha \beta) x^{4}-\beta x^{6}\right) \\
& \times f(x)=0 .
\end{aligned}
$$

Comparing (3) and (17) one can deduce that

$$
\begin{gathered}
p_{0}(x)=2\left(\alpha x+\beta x^{3}\right), \\
q_{0}(x)=\alpha-E+\left(1-\alpha^{2}+3 \beta\right) x^{2} \\
+(g-2 \alpha \beta) x^{4}-\beta x^{6} .
\end{gathered}
$$

By the aid of computer program, one can calculate the eigenvalues $E_{n}$ and the corresponding eigenfunctions $f(x)$ for a 
range of $g$ values, changing from 0.01 to 100 , using number of iterations $m=20,20,40,60,80,100,120$. The term "asymptotic" means the function approaching to a given value as the iteration number $m$ tends to infinity.

We present our results carried out for a range of $g$ values in Table 1 with 7 significant digits, and they are compared with those of supersymmetric perturbation approach by [40] and the ones computed numerically by [42]. In our calculations, we set $m=120, \alpha=4$, and $\beta=0$. It is observed that there is remarkable agreement in the whole range of values for all quantum states for different $g$ values with results of [42] except $g=100$.

We also present and compare our results for $g=0.1$ with those of Bacus et al. [39] in Table 2 with 20 significant digits. The function $f(x)$, for $g=0.1, n=5$ state for different values of $m$ is found as follow:

$$
\begin{aligned}
& m=20 \text {, } \\
& f(x)=x-1.3425 x^{3}-1.28331 x^{5}-0.249794 x^{7} \\
& +0.126907 x^{9}+0.0974402 x^{11}+0.0325753 x^{13} \\
& +0.00674249 x^{15}+0.000816039 x^{17}+O\left(x^{19}\right) \text {, } \\
& m=40 \text {, } \\
& f(x)=x-0.35941 x^{3}-0.998775 x^{5}-0.578949 x^{7} \\
& -0.161047 x^{9}-0.0125032 x^{11}+0.0088751 x^{13} \\
& +0.00478244 x^{15}+0.00143729 x^{17}+O\left(x^{19}\right), \\
& m=80 \text {, } \\
& f(x)=x-0.328321 x^{3}-0.980318 x^{5} \\
& -0.581291 x^{7}-0.167846 x^{9} \\
& -0.0162743 x^{11}+0.00762613 x^{13} \\
& +0.00449722 x^{15}+0.00139268 x^{17}+O\left(x^{19}\right), \\
& m=120 \text {, } \\
& f(x)=x-0.328321 x^{3}-0.980318 x^{5} \\
& -0.581291 x^{7}-0.167846 x^{9} \\
& -0.0162743 x^{11}+0.00762613 x^{13} \\
& +0.00449722 x^{15}+0.00139268 x^{17}+O\left(x^{19}\right) \text {. }
\end{aligned}
$$

For the first six states, the plot of the normalized wave functions for $g=0.1$ are given in Figure 2. As a last example, we consider the symmetric bistable potential defined in [43] as

$$
V(x)=x^{6}-2 x^{4}-2 x^{2}+1
$$

TABLE 3: Comparison of energy eigenvalues of the potential in (20). For ATEM results, number of iterations is set to $m=120$.

\begin{tabular}{lccc}
\hline$E_{\mathrm{ATEM}}$ & $E_{\mathrm{VSQM}}[43]$ & $E_{\mathrm{SDD}}[45]$ & $E_{\text {Exact }}[43]$ \\
\hline 0 & 0 & 0 & 0 \\
0.4229446 & 0.4238512 & 0.4229446 & 0.4229511 \\
2.314913 & 2.319117 & 2.314913 & 2.314925 \\
4.503779 & 4.571588 & 4.503779 & 4.503822 \\
7.175475 & 7.101165 & 7.175475 & 7.175509 \\
10.27788 & 9.861245 & 10.27789 & 10.27797 \\
13.75855 & 12.82074 & 13.75855 & 13.75861 \\
17.58421 & 15.95720 & 17.58420 & 17.58434 \\
21.72951 & 19.25351 & 21.72942 & 21.72951 \\
26.17305 & 22.69614 & 26.17370 & 26.17391 \\
\hline
\end{tabular}

Inserting the potential function into Schrödinger equation in (9) and using the ansatz wave function of the form defined in (16), one obtains

$$
\begin{aligned}
L(x)= & -f^{\prime \prime}(x)+2\left(\alpha x+\beta x^{3}\right) f^{\prime}(x) \\
& +\left(1+\alpha-2 E-\left(2+\alpha^{2}-3 \beta\right) x^{2}\right. \\
& \left.\quad-2(1+\alpha \beta) x^{4}+\left(1-\beta^{2}\right) x^{6}\right) f(x)=0 .
\end{aligned}
$$

Following the procedure given in (8) for $m=120, \alpha=4$, and $\beta=1$ yields the eigenvalues of the symmetric bistable potential in (20). We note here the selection of adjustable parameters $\alpha$ and $\beta$. Since we choose the ansatz wave function given in (16), the form of the superpotential for the partner potentials by supersymmetric quantum mechanics (SUSYQM) [44] is found to be

$$
W(x)=-\frac{\psi^{\prime}(x)}{\psi(x)},
$$

and the partner potentials $V_{ \pm}(x)$ are defined as

$$
V_{ \pm}(x)=W^{2}(x) \pm W^{\prime}(x) .
$$

Since the superpotential $W(x)$ obtained from (16) is

$$
W(x)=\alpha x+\beta x^{3},
$$

then the partner potential $V_{-}(x)$ is obtained as

$$
V_{-}(x)=\left(\alpha^{2}-3 \beta\right) x^{2}+2 \alpha \beta x^{4}+\beta^{2} x^{6}-\alpha .
$$

If $\alpha^{2}>3 \beta$, the potential (25) has one minimum. Investigating this one-minimum case one can select the adjustable parameters $\alpha=2$ and $\beta=1$ that lead to an increase in the iteration number $m$. Therefore, we select and set the parameters as $\alpha=4$ and $\beta=1$ in our calculations.

Our results are presented and compared with the values found by the variational supersymmetric method $\left(E_{\mathrm{VSQM}}\right)$ [43], state-dependent diagonalization method $\left(E_{\mathrm{SDD}}\right)$ [45], and by direct numerical integration $\left(E_{\text {Exact }}\right)$ [43], in Table 3. The ATEM results are in a very good agreement, by low percent errors, for all values of energies with the ones obtained by numerical calculation. For lower percent errors, the iteration number $m$ must be increased. 


\section{Conclusion}

An approximate method based on the asymptotic Taylor series expansion of a function and its fundamental features are presented. It is observed that the method is applicable for obtaining both eigenvalues and eigenfunctions of the Schrödinger-type equations. After applying the method to the one-dimensional harmonic oscillator potential, it is shown that the approach gives accurate results for eigenvalue problems of some certain type of bistable potentials. It is thought that the approach opens the way to the treatment of the Schrödinger equation including large class of potentials of practical interest. As a future study, the method can be developed and applied to the non-Hermitian systems and QES potentials. The direct application of the method to the Fokker-Planck equation for quasi exactly solvable bistable potentials when the drift coefficient has a nonpolynomial nature would also be interesting. Studies along this line are in progress.

\section{Acknowledgments}

This work is supported by the Research Fund Unit of Gaziantep University. Okan Ozer would like to thank Dr. G. Lévai (ATOMKI, Debrecen-Hungary) for supporting some papers used in this study, and it is a pleasure to thank Dr. R. Koc for valuable conversations and comments. The authors are also very much indebted to the referee(s) for useful comments and suggestions.

\section{References}

[1] E. Schrödinger, "Quantisierung als Eigenwertproblem," Annalen der Physik, vol. 79, no. 4, pp. 361-376, 1926.

[2] Y. P. Varshni, "Eigenenergies and oscillator strengths for the Hulthén potential," Physical Review A, vol. 41, no. 9, pp. 46824689, 1990.

[3] M. A. Nunez, "Accurate computation of eigenfunctions for Schrödinger operators associated with Coulomb-type potentials," Physical Review A, vol. 47, no. 5, pp. 3620-3631, 1993.

[4] C. Stubbins, "Bound states of the Hulthén and Yukawa potentials," Physical Review A, vol. 48, no. 1, pp. 220-227, 1993.

[5] P. Matthys and H. de Meyer, "Dynamical-group approach to the Hulthén potential," Physical Review A, vol. 38, no. 3, pp. 11681171,1988 .

[6] Y. P. Varshni, "Relative convergences of the WKB and SWKB approximations," Journal of Physics A, vol. 25, no. 21, pp. 57615777, 1992.

[7] G. A. Dobrovolsky and R. S. Tutik, "Regularization of the WKB integrals," Journal of Physics A, vol. 33, no. 37, pp. 6593-6599, 2000.

[8] A. Z. Tang and F. T. Chan, "Shifted $1 / N$ expansion for the Hulthén potential," Physical Review A, vol. 35, no. 2, pp. 911-814, 1987.

[9] R. K. Roychoudhury and Y. P. Varshni, "Shifted $1 / N$ expansion and exact solutions for the potential $V(r)=-Z / r+g r+\lambda r^{2}$," Journal of Physics A, vol. 21, no. 13, pp. 3025-3034, 1988.

[10] A. F. Nikiforov and V. B. Uvarov, Special Functions of Mathematical Physics, Birkhäuser, Basel, Switzerland, 1988.
[11] B. Gönül and K. Köksal, "A search on the Nikiforov-Uvarov formalism," Physica Scripta, vol. 75, no. 5, pp. 686-690, 2007.

[12] B. Gönül, O. Özer, Y. Cançelik, and M. Koçak, "Hamiltonian hierarchy and the Hulthén potential," Physics Letters A, vol. 275, no. 4, pp. 238-243, 2000.

[13] S. W. Qian, B. W. Huang, and Z. Y. Gu, "Supersymmetry and shape invariance of the effective screened potential," New Journal of Physics, vol. 4, article 13, 2002.

[14] A. K. Roy, "The generalized pseudospectral approach to the bound states of the Hulthén and the Yukawa potentials," Pramana Journal of Physics, vol. 65, no. 1, pp. 1-15, 2005.

[15] H. Ciftci, R. L. Hall, and N. Saad, "Asymptotic iteration method for eigenvalue problems," Journal of Physics A, vol. 36, no. 47, pp. 11807-11816, 2003.

[16] C. S. Lai and W. C. Lin, "Energies of the Hulthén potential for $l$ \# 0," Physics Letters A, vol. 78, no. 4, pp. 335-337, 1980.

[17] S. H. Patil, "Energy levels of screened Coulomb and Hulthen potentials," Journal of Physics A, vol. 17, no. 3, article 575, 1984.

[18] B. Roy and R. Roychoudhury, "The shifted $1 / N$ expansion and the energy eigenvalues of the Hulthen potential for 1 not $=0$," Journal of Physics A, vol. 20, no. 10, p. 3051, 1987.

[19] R. L. Greene and C. Aldrich, "Variational wave functions for a screened Coulomb potential," Physical Review A, vol. 14, no. 6, pp. 2363-2366, 1976.

[20] U. Myhrman, "A recurrence formula for obtaining certain matrix elements in the base of eigenfunctions of the Hamiltonian for a particular screened potential," Journal of Physics A, vol. 16, no. 2, pp. 263-270, 1983.

[21] A. Bechlert and W. Bhring, "Analytic model of atomic screening based on a generalised Hulthen potential," Journal of Physics B, vol. 21, no. 5, p. 817, 1988.

[22] R. Koç and S. Sayın, "Remarks on the solution of the positiondependent mass Schrödinger equation," Journal of Physics A, vol. 43, no. 45, Article ID 455203, 8 pages, 2012.

[23] M. Razavy, "An exactly soluble Schrödinger equation with a bistable potential," American Journal of Physics, vol. 48, no. 4, p. 285, 1980.

[24] Q.-T. Xie, "New quasi-exactly solvable double-well potentials," Journal of Physics A, vol. 45, no. 17, Article ID 175302, 7 pages, 2012.

[25] B. U. Felderhof, "Diffusion in a bistable potential," Physica A, vol. 387, no. 21, pp. 5017-5023, 2008.

[26] H. Ciftci, O. Ozer, and P. Roy, "Asymptotic iteration approach to supersymmetric bistable potentials," Chinese Physics B, vol. 21, no. 1, Article ID 010303, 2012.

[27] T. Barakat, "The asymptotic iteration method for the eigenenergies of the anharmonic oscillator potential $V(x)=A x 2 \alpha+B x^{2}$," Physics Letters A, vol. 344, no. 6, pp. 411-417, 2005.

[28] A. J. Sous, "Solution for the eigenenergies of sextic anharmonic oscillator potential $V(x)=A_{6} x^{6}+A_{4} x^{4}+A_{2} x^{2}$," Modern Physics Letters $A$, vol. 21, no. 21, pp. 1675-1682, 2006.

[29] T. Barakat and O. M. Al-Dossary, "The asymptotic iteration method for the eigenenergies of the asymmetrical quantum anharmonic oscillator potentials $V(x)=\sum_{j=2}^{2 \alpha} A_{j} x^{j}$," International Journal of Modern Physics A, vol. 22, no. 1, pp. 203-212, 2007.

[30] T. Brook, Methodus Incrementorum Directa et Inversa (Direct and Reverse Methods of Incrementation), impensis Gulielmi Innys, London, UK, 1715.

[31] D. J. Struik, A Source Book in Mathematics, Translated into English, University Press, Cambridge, Mass, USA, 1969. 
[32] Wolfram Research, Mathematica, Version 8. 0, Wolfram Research, Champaign, Ill, USA, 2010.

[33] J. P. Boyd, "The devil's invention: asymptotic, superasymptotic and hyperasymptotic series," Acta Applicandae Mathematicae, vol. 56, no. 1, pp. 1-98, 1999.

[34] C. S. Hsue and J. L. Chern, "Two-step approach to one-dimensional anharmonic oscillators," Physical Review D, vol. 29, no. 4 , pp. 643-647, 1984.

[35] G. P. Flessas, R. R. Whitehead, and A. Rigas, "On the $\alpha x^{2}+\beta x^{4}$ interaction," Journal of Physics A, vol. 16, no. 1, pp. 85-97, 1983.

[36] R. A. Bonham and L. S. Su, "Use of Hellmann-Feynman and hypervirial theorems to obtain anharmonic vibration-rotation expectation values and their application to gas diffraction," Journal of Chemical Physics, vol. 45, no. 8, p. 2827, 1996.

[37] M. Reed and B. Simon, Methods of Modern Mathematical Physics, IV: Analysis of Operators, Academic Press, New York, NY, USA, 1978.

[38] N. Aquino, "Asymptotic series for the quantum quartic anharmonic oscillator," Journal of Mathematical Chemistry, vol. 18, no. 2, pp. 349-357, 1995.

[39] B. Bacus, Y. Meurice, and A. Soemadi, "Precise determination of the energy levels of the anharmonic oscillator from the quantization of the angle variable," Journal of Physics A, vol. 28, no. 14, pp. L381-L385, 1995.

[40] B. Gönül, N. Çelik, and E. Olğar, "A new algebraic approach to perturbation theory," Modern Physics Letters A, vol. 20, no. 22, pp. 1683-1694, 2005.

[41] R. Koc and E. Olgar, 2010, Preprint math-ph/1008.0697.

[42] K. Banerjee, "General anharmonic oscillators," Proceedings of the Royal Society of London A, vol. 364, no. 1717, pp. 265-275, 1978.

[43] G. R. P. Borges, E. D. Filho, and R. M. Ricotta, "Variational supersymmetric approach to evaluate Fokker-Planck probability," Physica A, vol. 389, no. 18, pp. 3892-3899, 2010.

[44] A. Khare and Y. P. Varshni, "Is shape invariance also necessary for lowest order supersymmetric WKB to be exact?" Physics Letters A, vol. 142, no. 1, pp. 1-4, 1989.

[45] F. So and K. L. Liu, "Study of the Fokker-Planck equation of bistable systems by the method of state-dependent diagonalization," Physica A, vol. 277, no. 3, pp. 335-348, 2000. 


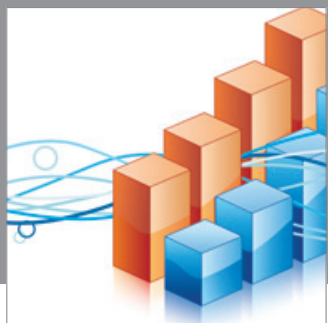

Advances in

Operations Research

mansans

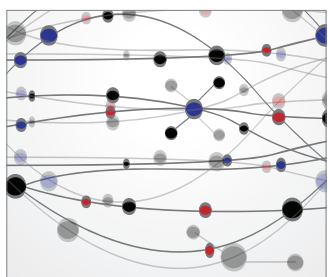

The Scientific World Journal
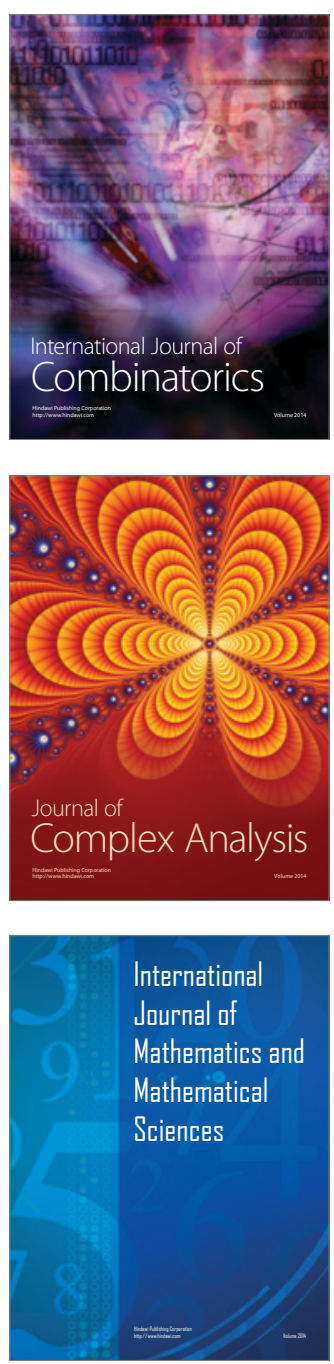
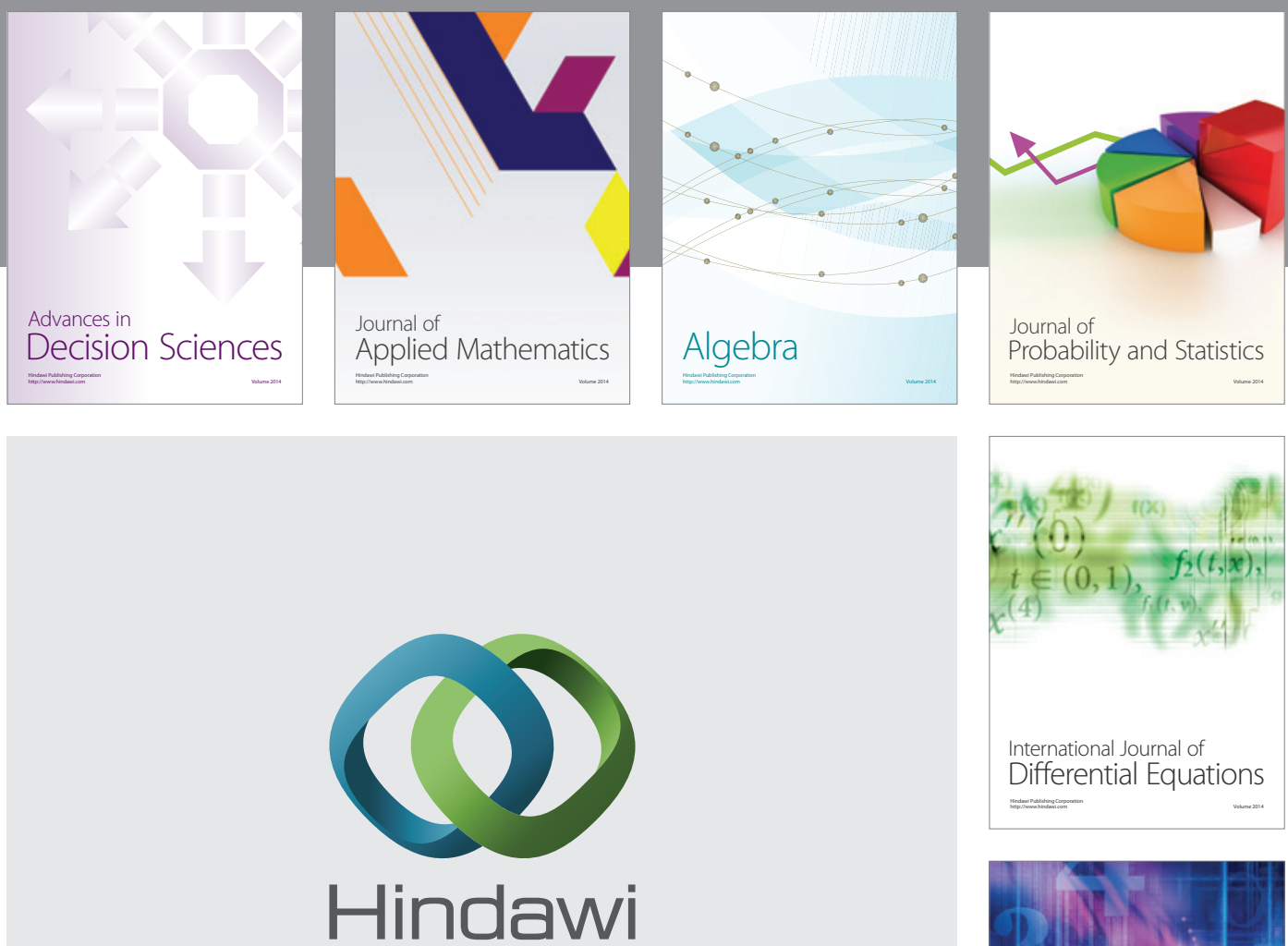

Submit your manuscripts at http://www.hindawi.com
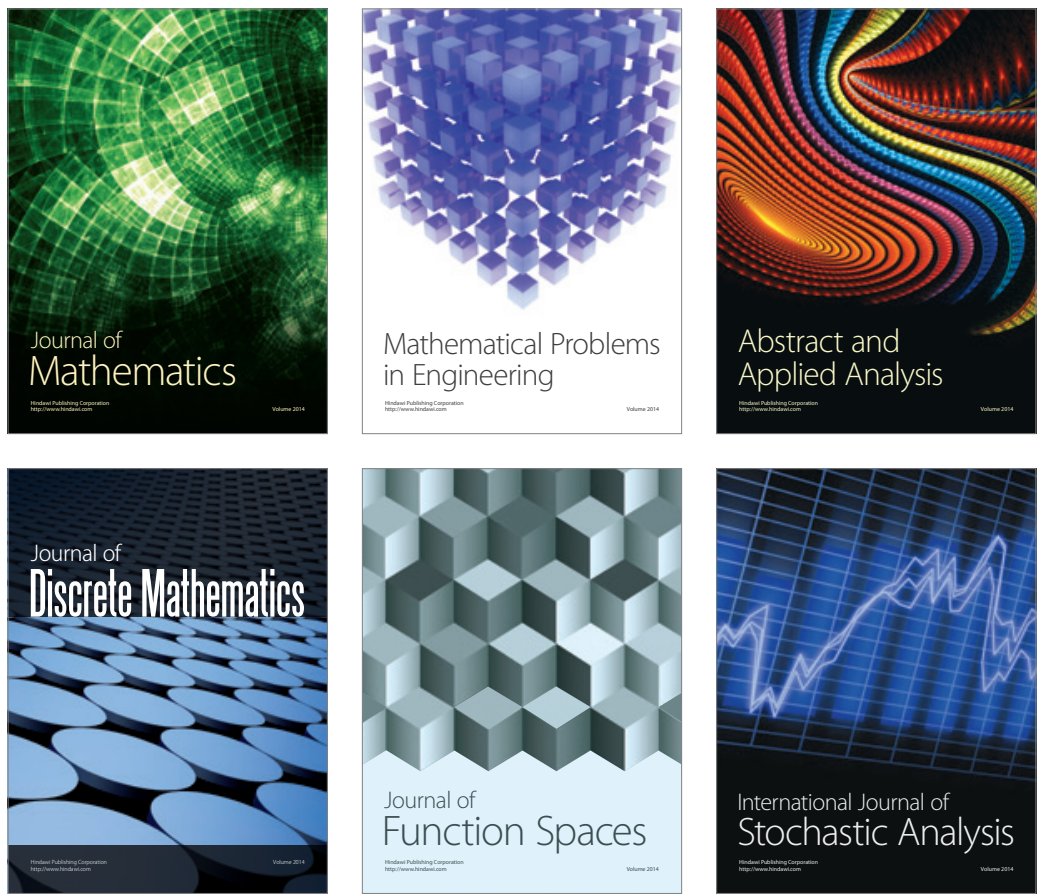

Journal of

Function Spaces

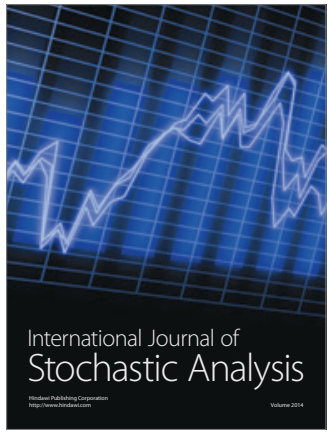

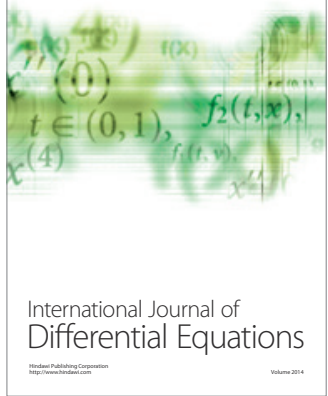
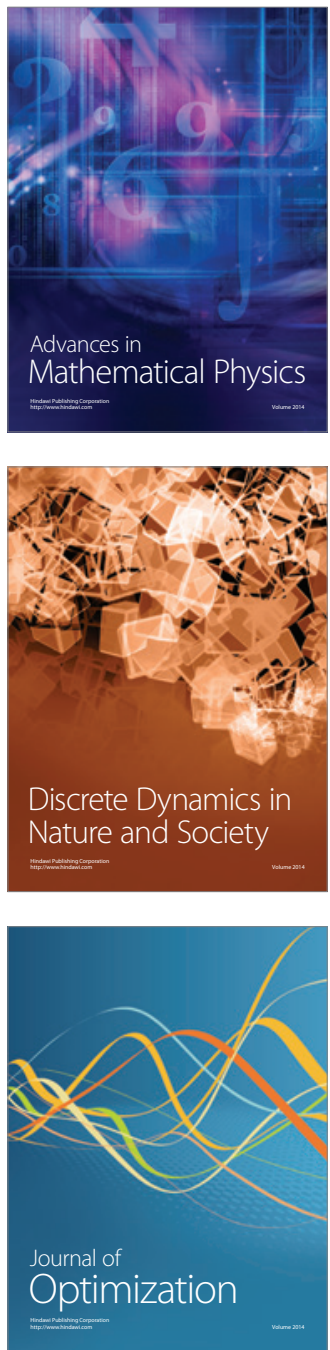